\title{
Klein Nishina Differential Equation for the Selection of Radiation Shielding Material (C, AL, Fe, and Zn) on the Basis of Attenuation and Cross sectional Area
}

\author{
Saddam Husain Dhobi, Santosh Kumar Das, and Kishori Yadav
}

\section{ABSTRACT}

\begin{abstract}
On studying the Electronic and Atomic Cross sectional area for low atomic masses (Carbon, Aluminum, Iron and Zinc) using Klien-Nishina differential equation. The atomic cross section among these elements for same energy of incidence photon the atomic cross section area found on order of Carbon $<$ Aluminum $<$ Iron $<$ Zinc. This show with increasing atomic number and mass the cross section area of material goes increase. But the mass attenuation goes decrease with increasing in mass and number of materials made up of high atomic weight and number. This is clearly seen in Fig. 2 and Fig. 3. Therefore, among these elements protective material is made up of Carbon has more safety than other (Al, Fe, Zn).
\end{abstract}

Keywords: Cross sectional Area, Low atomic masses, Photon, Material, Elements etc.
Published Online: January 18, 2021.

ISSN: $2684-4451$

DOI :10.24018/ejphysics.2021.3.1.38

\section{S. H. Dhobi*}

Innovative Ghar Nepal, Lalitpur, Nepal. Robotics Academy of Nepal, Lalitpur, Nepal.

Department of Physics, Tribhuvan University, Kathmandu, Nepal.

(e-mail: saddam@ ${ }^{@}$ ran.edu.np)

(e-mail: saddamdhobe@ ${ }^{@ a m i l . c o m) ~}$ Santosh Kumar Das

Department of Physics, Tribhuvan University, Kathmandu, Nepal.

Kishori Yadav

Department of Physics, Tribhuvan University, Kathmandu, Nepal.

*Corresponding Author

\section{INTRODUCTION}

X-ray hazards recognized with the discovery but depend on dose limitation by time, distance, and shielding. For the protection from X-rays, Taylor (1979) effort for guidance of $\mathrm{X}$-ray with British and German for x-ray shielding [1]. The characteristic of carbon nanotubes used for radiation shielding capability. For Carbon nanotubes the linear and mass attenuation coefficient in low energy (up to $30 \mathrm{keV}$ ). For the improvement of properties of shielding different carbonbased polymeric composites is current research [2].

The enhancing of shielding effect with graphene nanocomposites was demonstrated with thin films of ferroelectric. For this X-ray beams energies of 6.9 and 8.1 $\mathrm{keV}$, respectively demonstrated and found attenuate $82.9 \%$ and $48.5 \%$. The enhanced attenuation appears in the region where photoelectric absorption starts to predominate against coherent and incoherent scattering, toward lower energies. Lightweight, very thin, and highly efficient PVDF/GO radiopaque films can be now manufactured, aiming to minimize patient skin injuries in high-dose interventional radiology procedures [3].

Graphene and GNPs is expected for high intrinsic electric conductivity with great improvement for the microwave absorption of their composites [4]. Photoelectric absorption phenomena are dominant at lower energy $(500 \mathrm{keV})$ and for shielding materials high atomic number elements like tungsten, lead and bismuth are used. Compton scattering is predominant in rage high energies $(500-1500 \mathrm{keV})$ and for shielding low atomic number element and materials like water, Aluminum and Iron is used. Pair production is dominant for very high energy radiation $(>1500 \mathrm{keV})$. The attenuation unit is $\mathrm{cm}^{2} / \mathrm{g}$, material density and thickness help to achieve desired level of attenuation. The attenuation of gamma and X-rays dependent upon the density of the shielding material [5].

Calcium oxalate and hydroxyapatite attenuating properties is equivalent to aluminum attenuating properties but depend upon thickness. Hydroxyapatite has high attenuating properties than calcifications and aluminum [6]. The X-ray attenuation induced with carbon nanotubes was observed with higher significantly $(>100 \%)$ than pyrolytic graphite and fullerenes. The attenuation coefficient of carbon nanotubes increased with reducing the sample thickness and attenuation enhanced up to $70 \%$ for X-rays [7].

In radiological physics, Photoelectric Absorption, Compton Scattering and Pair production, phenomena are dominant processes [8]. The attenuating properties of $\mathrm{Pb}$ and non- $\mathrm{Pb}$ based shielding materials are studied and used for radiation attenuation. In garment materials pure $\mathrm{Pb}$ with 0.25 and $0.5 \mathrm{~mm}$ lead equivalent is used. Not a single or combination material optimum shielding for all energy ranges but for a particular energy range one can select a single or combined material [9].

Millions of X-ray examinations, both diagnostic and interventional, nuclear medicine procedures and radiotherapy treatments are performed annually worldwide. Radiology is an essential component of patient management, as many 
patients have multiple investigations using ionizing radiation, particularly computed tomography (CT), and some go on to have X-ray guided treatment or radiotherapy. The radionuclides used in medicine increases from simple to complex radiotherapy with huge benefits for health worker and patients [10].

There are two general classifications of Radiation shielding are thermal and biological. In thermal shielding is used to dissipate excessive heat while biological shielding reduce radiation. The combination of both help to reduce the problem caused by heat and radiation.

Lead is one of the best attenuation materials with favorable density, cost-effective, easily availability. Currently, researchers and scientist are developing the material for shielding which substitute lead because lead is heavy, causes human poisoning, and hazardous waste. Therefore, researchers and scientists are working to replace lead with bismuth, tungsten, barium, tin, cerium, and antimony. This is due to process the material into a thin film, a base material with excellent flexibility is preferred [11-15]. The energy of photons like X-rays and gamma rays is shield with the lower the atomic number with minimal thickness. These radiations are ionizing radiation with wide applications like medical diagnosis, cancer treatment, food preservation and nuclear energy. With the benefit it has also adverse effect on human health when they take overdose for long time [16].

The absorbed dose of radiation energy refers to energy incidence and absorbed by tissues per unit of mass. The effective dose of radiation is used by applying a radiation weighting factor and a tissue-weighting factor to the absorbed dose, which is measured in Sieverts (Sv). Therefore, a standard effective dose of ration for different part of body is different used in medical diagnostic.

\section{REVIEW}

A report from 1991 to 1996 show 2.4 billion are diagnostic medical examinations worldwide and among them 250 million are children below 15 years of age. Another report from 1997 to 2007 show 3.6 billion are diagnostic medical examinations and among them 350 million are children below 15 years of age reported by UNSCEAR in $2000 \& 2010$. Among these the Chest radiograph is $40 \%$ and $9 \%$ of chest radiographs are performed in children from middle income country.

The average exposure of radiation from all sources is about $3 \mathrm{mSv} /$ year, worldwide among then about $2.4 \mathrm{mSv} / \mathrm{year}$ is natural background radiation. About $0.6 \mathrm{mSv} / \mathrm{year}$ are from medical imaging and therapeutics which is artificial [17].

Klein-Nishina scattering cross section:

$$
\frac{d \sigma}{d \Omega}=r_{e}^{2}\left(\frac{k^{\prime}}{k_{0}}\right)^{2}\left(1+\cos ^{2} \theta\right)
$$

Here $k^{\prime} / k_{0}$ is calculated with angle $\theta$ using the Compton scattering formula and $r_{\mathrm{e}}$ is known as the classical electron

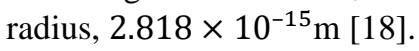

The modified form of above equation is:

$$
\frac{d \sigma}{d \Omega}=\frac{r_{e}^{2}}{2}\left(\frac{k^{\prime}}{k_{0}}\right)^{2}\left[\left(1+\cos ^{2} \theta\right)+\frac{\left(k_{0}-k^{\prime}\right)^{2}}{k_{0} k^{\prime}}\right]
$$

Clearly this term is positive, thus increasing the expected differential cross section at all scattering angles greater than 0 . Its effect is evidently greatest for large incident photon energies and for large scattering angles by substituting $2-$ $\sin ^{2} \theta$ for $1+\cos ^{2} \theta$, expanding the numerator of the extra term and simplifying as"

$$
\frac{d \sigma}{d \Omega}=\frac{\mathrm{r}_{\mathrm{e}}^{2}}{2}\left(\frac{k^{\prime}}{k_{0}}\right)^{2}\left(\frac{k^{\prime}}{k}+\frac{k_{0}}{k^{\prime}}-\sin ^{2} \theta\right)
$$

The rates for the scattering of high-energy photons depend upon the various angles $\theta$ of incidence of photon and. The scattering of photons in Compton Effect characterize the physical properties and analyzed to predict uncertainty of Compton photons. This effect optimized the geometrical parameters for Compton scattering analysis, based on energy and incident flux X-ray photons [19].

The atomic orbital structure and in specific the momentum distributions of the atomic electrons are well known to become apparent in Compton scattering and positron annihilation in matter. The cross section of Compton scattering for a free target electron at rest was described by Klein Nishina (KN) differential equation. In case of Compton scattering, this is particularly true at low photon energies and for heavy nuclei (with tightly bound electrons). The wide application of Compton scattering take place where photon transport is important, notably dosimetry, shielding, photon detectors, medical linacs, positron emission tomography (PET) and dose monitoring with prompt photons [20]-[23].

Compton scattering is also called incoherent scattering which is the dominant interaction in $\mathrm{Kev}$ to $5 \mathrm{MeV}$ energy ranges. If the momentum transfer of a photon is large compared to the electron binding energies of the target atoms, Compton scattering can be described with good accuracy in the free-electron approximation. For a free electron at rest, the scattering cross section is given by the well-known $\mathrm{KN}$ formula [24].

The photons with energy undergoing absorption or scattering when interact with material. The linear attenuation coefficient is given as $\mu\left(\mathrm{cm}^{-1}\right)$, where $\mu$ dependent on density of material's $\rho\left(\mathrm{g} \mathrm{cm}^{-3}\right)$. The mass attenuation coefficient is given as $\mu / \rho\left(\mathrm{cm}^{2} \mathrm{~g}^{-1}\right)$ which relate both density and linear attenuation. The major interaction processes, incoherent (Compton) scattering, coherent (Rayleigh) scattering and atomic photo effect absorption take place with photon energies below $1 \mathrm{MeV}$. Above $1 \mathrm{MeV}$, pair production and triplet production phenomena take place, while with above $5 \mathrm{MeV}$ photonuclear absorption take place [25]. An experiment with free electron Compton scattering cross-section was done with the help of Klein-Nishina with photon energy of $662 \mathrm{keV}$ and goes incoherently scattering through angles from $0-120^{\circ}$. The observation shows the energy of scattered photon ray decreases as the scattering angles increase and differential scattering cross-section as a function of $\theta$ was related [26].

As said, to insert images in Word, position the cursor at the insertion point and either use Insert | Picture | From File or 
copy the image to the Windows clipboard and then Edit Paste Special | Picture (with "Float over text" unchecked).

The authors of the accepted manuscripts will be given a copyright form and the form should accompany your final submission.

\section{Methodology}

When a beam of monochromatic photons of incident energy $E_{0}$, in a photon flux $\Phi_{0}$, interacts with a sample of homogeneous material of thickness $x$, the emergent flux $\Phi$ can be written as the Beer-Lambert equation:

$$
\Phi=\Phi_{0} e^{-\mu x}
$$

where $\mu\left(\mathrm{cm}^{-1}\right)$ is the linear attenuation coefficient for a material of physical density $\rho\left(\mathrm{gcm}^{-3}\right)$ and atomic number $\mathrm{Z}$ and $\mathrm{x}$ is the thickness of the sample. For energy values over $10 \mathrm{keV}$, when the molecular binding energy is low, it is reasonable to assume that $\mu\left(\mathrm{cm}^{-1}\right)$ is directly proportional to the physical density:

$$
\Phi=\Phi_{0} e^{-\left(\frac{\mu}{\rho}\right) \rho x}
$$

where $\frac{\mu}{\rho}\left(\mathrm{cm}^{2} \mathrm{~g}^{-1}\right)$ is the mass attenuation coefficient, which is proportional to the total cross-section per electron $\sigma_{t}^{e}(E)$ cm2/electron as:

$$
\frac{\mu}{\rho}=\frac{\sigma_{t}^{e}(E) Z N_{A}}{A}
$$

where $\mathrm{N}_{\mathrm{A}}$ is the Avogadro's number(6.02 $\times 10^{23}$ atom/ $\mathrm{mol}), \mathrm{Z}$ is the atomic number and $\mathrm{A}$ is the material atomic mass, hence linear attenuation coefficient is given as:

$$
\mu=\frac{\rho \sigma_{t}^{e}(E) Z N_{A}}{A} \text { or } \mu=\sigma_{t}^{e}(E)\left(\frac{\rho Z N_{A}}{A}\right)
$$

where $\delta_{e}$ electron density and represents the number of electrons per cubic centimeter is given as:

$$
\delta_{e}=\frac{\rho Z N_{A}}{A}
$$

Therefore, on substituting the value we get:

$$
\mu=\sigma_{t}^{e}(E) \delta_{e}
$$

On the other hand, in a general way the total cross-section $\sigma_{t}^{e}(E)$ can be written as the addition of the different crosssections' contributions related to the manners of radiation interaction with the matter, given by:

$$
\sigma_{t}^{e}(E)=\sigma_{p h}^{e}(E)+\sigma_{C}^{e}(E)+\sigma_{R}^{e}(E)+\sigma_{N I}^{e}(E)
$$

where, $\sigma_{p h}^{e}(E)=$ Cross section for Photoelectric effect. $\sigma_{C}^{e}(E)=$ Cross section for Compton Scattering. $\sigma_{R}^{e}(E)=$ Cross section for Rayleigh scattering. $\sigma_{N I}^{e}(E)=$ Cross section for Nuclear Interaction.
With the energy range of photon 10 to $300 \mathrm{Kev}$ the probability of occurrence of Rayleigh scattering and the nuclear interactions almost zero. Therefore, the above equation become:

$$
\sigma_{t}^{e}(E) \approx \sigma_{p h}^{e}(E)+\sigma_{C}^{e}(E)
$$

The attenuated in the path of photon beam, resulting the count of scattered photons is given as:

$S=\Phi_{0} \exp \left(-\int_{x_{1}} \mu_{1}(x) d x-\int_{x_{2}} \mu_{2}(x) d x\right) \frac{d_{e} \sigma^{K N}}{d \Omega} \delta_{e} d V d \Omega$

where $\mu_{1}$ and $\mu_{2}$ are the linear attenuation coefficients in the paths between source and sample $\left(x_{1}\right)$ and sample and detector $\left(x_{2}\right)$, respectively, $d V$ is the scattering volume and $d \Omega$ is the detector field of view.

The generalized Klein-Nishina differential formula for Compton scattering with a finite train of pulses also applicable to classical Thomson scattering. The number of scattering photons generated into a given solid angle $d \Omega$ is:

$\frac{d N_{\text {scat }}}{d \Omega}=\int_{-\infty}^{\infty} \frac{\epsilon_{0} c}{2 \pi} \frac{\left|E_{\chi}(\omega)\right|^{2}}{\hbar|\omega|} \frac{d \sigma}{d \Omega} d \omega$

And because the scattered photon has energy $\hbar \omega^{\prime}$, the total scattered energy is:

$\frac{d U_{r}}{d \Omega}=\int_{-\infty}^{\infty} \frac{\epsilon_{0} c}{2 \pi}\left|E_{x}(\omega)\right|^{2} \frac{\omega \prime}{\omega} \frac{d \sigma}{d \Omega} d \omega$

\section{A. Klien-Nishina Differential Cross Section with Atomic Number}

The dominant scattering process for $511 \mathrm{Kev}$ photons is Compton scattering, where the incident gamma-ray strikes an atomic electron producing atomic ionization. The incident photon will scatter through an angle $\theta$ determined by the KIein-Nishina differential cross section equation:

$$
\begin{aligned}
& \left(\frac{d \sigma}{d \Omega}\right)_{a}=Z r_{e}^{2}\left(\frac{1}{1+\alpha(1-\cos \theta)}\right)^{2} \frac{\left(1+\cos ^{2} \theta\right)}{2}(1+ \\
& \left.\frac{\alpha^{2}(1-\cos \theta)^{2}}{\left(1+\cos ^{2} \theta\right)[1+\alpha(1-\cos \theta)]}\right)
\end{aligned}
$$$$
\left(\frac{d \sigma}{d \Omega}\right)_{a}=\frac{Z r_{e}^{2}}{2}\left(\frac{1}{1+\alpha(1-\cos \theta)}\right)^{2}\left(\left(1+\cos ^{2} \theta\right)+\right.
$$$$
\left.\frac{\alpha^{2}(1-\cos \theta)^{2}\left(1+\cos ^{2} \theta\right)}{\left(1+\cos ^{2} \theta\right)[1+\alpha(1-\cos \theta)]}\right)
$$

$$
\begin{aligned}
& \left(\frac{d \sigma}{d \Omega}\right)_{a}=\frac{Z r_{e}^{2}}{2}\left(\frac{1}{1+\alpha(1-\cos \theta)}\right)^{2}\left(\left(1+\cos ^{2} \theta\right)+\right. \\
& \left.\frac{\alpha^{2}(1-\cos \theta)^{2}}{[1+\alpha(1-\cos \theta)]}\right)
\end{aligned}
$$

$\left(\frac{d \sigma}{d \Omega}\right)_{a}=\frac{Z r_{e}^{2}}{2}\left(\frac{1}{1+\alpha(1-\cos \theta)}\right)^{2}\left(\left(1+\cos ^{2} \theta\right)+\right.$

$\left.\frac{\alpha^{2}(1-\cos \theta)^{2}}{[1+\alpha(1-\cos \theta)]}\right)$

This equation is a differential atomic cross sectional area equation for K-N. 
Also, the total K-N cross section per atom can be written as:

$\sigma_{a}=2 \pi \int_{0}^{\pi}\left(\frac{d \sigma}{d \Omega}\right)_{a} \sin \theta d \theta$

where $\theta$ is scattering angle overall photons.

Now from (3) and (4), we get:

$\sigma_{a}=2 \pi \int_{0}^{\pi} \frac{z r_{e}^{2}}{2}\left(\frac{1}{1+\alpha(1-\cos \theta)}\right)^{2}\left(\left(1+\cos ^{2} \theta\right)+\right.$

$\left.\frac{\alpha^{2}(1-\cos \theta)^{2}}{[1+\alpha(1-\cos \theta)]}\right) \sin \theta d \theta$

On solving the total $\mathrm{KN}$ cross section per atom is obtained as:

$\sigma_{a}=Z 2 \pi r_{0}^{2}\left\{\frac{1+\alpha}{\alpha^{2}}\left[\frac{2(1+\alpha)}{1+2 \alpha}-\frac{\ln (1+2 \alpha)}{\alpha}\right]+\frac{\ln (1+2 \alpha)}{2 \alpha}-\frac{1+3 \alpha}{(1+2 \alpha)^{2}}\right\}$

Since Klein-Nishina atomic cross-sections are obtained by multiplying electronic cross-sections with charge number $\mathrm{Z}$ of each element that is $\sigma_{a}=Z . \sigma_{e}$, therefore from equation (5), the electronic cross-sectional area for $\mathrm{KN}$ is:

$\sigma_{e}=2 \pi r_{0}^{2}\left\{\frac{1+\alpha}{\alpha^{2}}\left[\frac{2(1+\alpha)}{1+2 \alpha}-\frac{\ln (1+2 \alpha)}{\alpha}\right]+\frac{\ln (1+2 \alpha)}{2 \alpha}-\frac{1+3 \alpha}{(1+2 \alpha)^{2}}\right\}$

where $r_{0}=2.818 \mathrm{fm}$ is the classical electron radius, $\mathrm{Z}$ is the nuclear charge of the target molecule and $\alpha=\frac{E}{m_{e} c^{2}}=$ $\frac{h f}{0.511 \mathrm{MeV}}$ (Knoll, 1989) [27], [28].

On putting the value of $\sigma_{e}$ in $\frac{\mu}{\rho}=\frac{\sigma_{e} Z N_{A}}{A}$ we get:

$\frac{\mu}{\rho}=2 \pi r_{0}^{2}\left(\frac{Z N_{A}}{A}\right)\left\{\frac{1+\alpha}{\alpha^{2}}\left[\frac{2(1+\alpha)}{1+2 \alpha}-\frac{\ln (1+2 \alpha)}{\alpha}\right]+\frac{\ln (1+2 \alpha)}{2 \alpha}-\right.$

$\left.\frac{1+3 \alpha}{(1+2 \alpha)^{2}}\right\}$

Therefore, this equation gives mass attenuation coefficient in term of $\mathrm{KN}$ parameters and known as Compton mass attenuation coefficient is calculated using, $\frac{\mu}{\rho}=N_{A} Z \frac{\sigma_{e}}{A}$. Where $\mathrm{N}_{\mathrm{A}}$ is the Avogadro's number (6.02 $\times 10^{23}$ atom/ $\mathrm{mol}$ ), $\mathrm{Z}$ is the atomic number and $\mathrm{A}$ is the material atomic mass.

Also, the linear attenuation coefficient in term of $\mathrm{KN}$ parameters is given as can be given by:

$\mu=2 \pi r_{0}^{2} \rho\left(\frac{Z N_{A}}{A}\right)\left\{\frac{1+\alpha}{\alpha^{2}}\left[\frac{2(1+\alpha)}{1+2 \alpha}-\frac{\ln (1+2 \alpha)}{\alpha}\right]+\frac{\ln (1+2 \alpha)}{2 \alpha}-\right.$

$\left.\frac{1+3 \alpha}{(1+2 \alpha)^{2}}\right\}$

The corresponding K-N cross section per unit mass, $\frac{\sigma}{\rho}$ is also called the Compton mass attenuation coefficient [28].

$\frac{d_{e} \sigma^{K N}(E)}{d \Omega}=\frac{r_{0}^{2}}{2}\left[\frac{\left(1+\cos ^{2} \theta\right)}{(1+\alpha(1-\cos \theta))^{2}}\left[1+\frac{\alpha^{2}(1-\cos \theta)^{2}}{\left(1+\cos ^{2} \theta\right)(1+\alpha(1-\cos \theta))}\right]\right]$

where $r_{0}$ is the classical electron radius $(2.818 \times$ $10^{-13} \mathrm{~cm}$ ) [29].

\section{RESUlt AND DisCUSSION}

The study of the cross-section area for the protection of health workers and patients from X-rays is important during diagnosis, treatment, and therapy. This work considers low atomic materials like carbon, aluminum, Iron, and Zinc because the properties of the material made from these elements have flexibility, lighter weight, mechanical strength, etc. Also, this element has very fewer hazards and easily available with cost-effectiveness. The lighter atomic masses have a low density, and the health worker wears them for a long time without any difficulties. Therefore, these elements material is one of the best options for health workers and their protection from X-rays.

The Klein-Nishina Electronic Cross section with the energy of $0.200 \mathrm{MeV}$ to $10 \mathrm{MeV}$ is shown below in Fig. 1 . The representation shows that with increasing the energy of incidence photon on the protective material wear by a health worker. The electronic cross section area of atoms, from which protective material is made decreases. The increase in the electronic cross section area, increase the bulk volume of the protective material used by health workers. The electronic cross section area using Klein-Nishina parameters for protective material made of Carbon, Aluminum, Iron and Zinc.

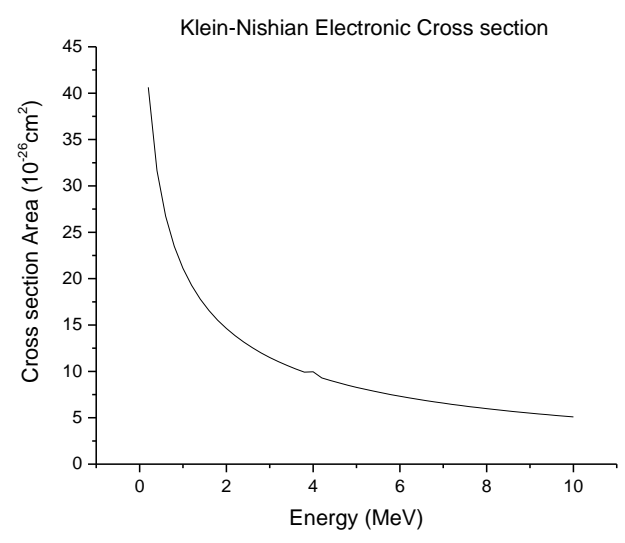

Fig. 1. Klein Nishina Electronic Cross Section Area.

On incidence, the $\mathrm{x}$-rays on the protective material made of up these elements go on Compton and Photoelectric effect. These both effects cause the reduction of the energy of photon when incidence on it. Therefore, the energy of photons goes to reduce and protect tissue or cells of health workers exposed to such radiation.

The atomic cross section of Klein-Nishina $(\mathrm{KN})$ for elements can be used for the protection of X-ray. The selected elements for our research work are Carbon, Ammonium, Zinc, and Iron. With the incidence of fixed energy photon on the atoms of each element, the atomic cross section using Klein-Nishina is represented in Fig. 2. Since the X-ray energies ranges are different, therefore in our study $0.200 \mathrm{MeV}$ to $10 \mathrm{MeV}$ are used. This is because this energy goes for the phenomena of Photoelectric effect and Compton Effect and reduced the energy of X-ray.

Among the selection element for the same energy incidence, the atomic cross section area of carbon atom is less than other. For the same case, Zinc has a high cross section area than others. Therefore, the bulk volume of the material made up of Zinc element goes increase and is maximum than 
other, while Carbon is less. Since the energy for all elements that are considered in this work equal therefore the to select the material for protective material Carbon is best. The KN atomic cross section area increases with increasing atomic weight, atomic number, and energy of photon therefore to improve the bulk expansion of the material low atomic weight is best.

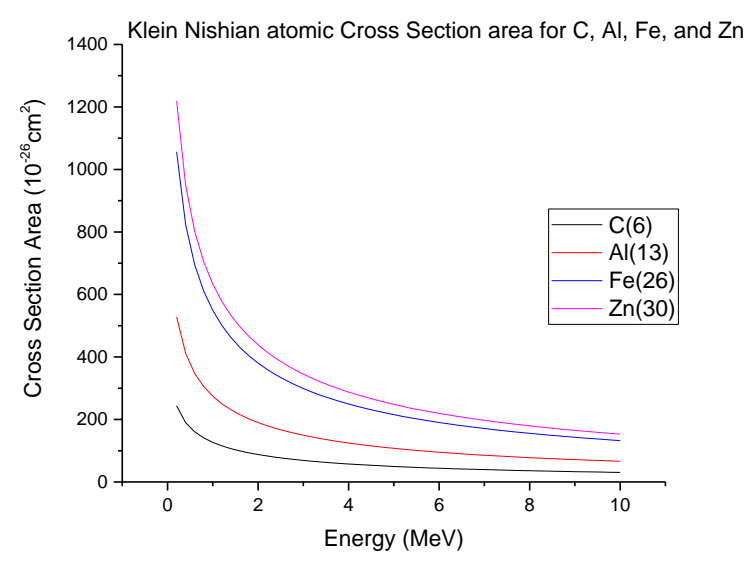

Fig. 2. Klein Nishina Atomic Cross section for material made up of C, Al, $\mathrm{Fe}, \mathrm{Zn}$.

The Mass Attenuation coefficient for Klein Nishina Cross section of considering element is shown in Fig. 3. The attenuation of carbon, Aluminum, Iron, and Zinc have the same behavior with X-ray of energy $0.200 \mathrm{MeV}$ to $10 \mathrm{MeV}$. The attenuation coefficient among considered elements is high for carbon and less for Zinc. This means the absorption of the X-ray with Carbon element is high while for Zinc is less. Therefore, based on the attenuation coefficient, the selection of protective material for X-ray Carbon is again best.

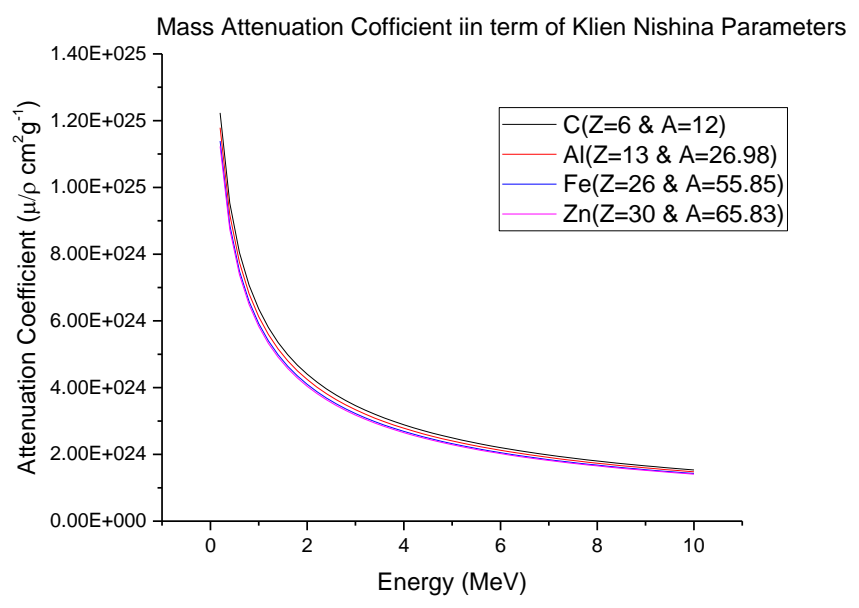

Fig. 3. Mass Attenuation Coefficient for Klien Nishina Cross section for material made up of $\mathrm{C}, \mathrm{Al}, \mathrm{Fe}, \mathrm{Zn}$.

As mass Attenuation Coefficient increase the absorption of photon increase that causes the energy loss of the photon and then the effect on the patient and health work is less or safe. On comparison of the Attenuation coefficient, the attenuation coefficient of increasing order as $\mathrm{C}>\mathrm{Al}>\mathrm{Fe}>\mathrm{Zn}$. Therefore, the absorption or energy of photon goes decreases more on the material made up of $\mathrm{C}$ rather than $\mathrm{Al}, \mathrm{Zn}$, and $\mathrm{Fe}$.
The benefit of carbon among this selected material is lighter weight and strength. This is because the exposure person under the radiation who wears protective equipment or cloth in the radiation room with carbon material goes more protected than the protective equipment of Aluminum, Zinc, and Iron.

\section{CONCLUSION}

Klein Nishina, electronic and cross section area increases with a decrease in the energy of incidence photon and vice versa. The energy for our study ranges from $0.200 \mathrm{MeV}$ to 10 $\mathrm{MeV}$, this energy of photon also goes to Photoelectric effect and Compton Effect. The wearing of protective equipment for health workers who are exposed to energy is made up of such type of materials which goes under Photoelectric and Compton Effect. This is because such material reduces the energy of incidence photon and protects the health worker from radiation. Among the consider element (Carbon, Aluminum, Zinc and Iron) for our study, Carbon is best because the attenuation coefficient is high.

If health workers wear protective equipment or cloths made up of Carbon, they get more protection than the equipment made up of Iron, Zinc, and Aluminum. The nature of Klein-Nishina atomic Cross section for Carbon, Iron, Zinc, and Aluminum same but cross section area of Carbon<Aluminum<Iron<Zinc. Therefore, for the same energy of incidence the cross section area is different. The expansion of the material is different from the incidence of the photon that is the expansion of Carbon<Aluminum<Iron<Zinc. The expansion of these cross sections results in the expansion of the bulk of material from which they were made. Hence the resultant expansion of material (Protective Equipment) made up of Carbon goes less expansion than others.

\section{ACKNOWLEDGMENT}

We would like to thank all members of Innovative Ghar Nepal, Department of Physics (Patan Multiple Campus, Tribhuvan University, Nepal), Robotics Academy of Nepal for their kind support and providing a peaceful environment during our work.

\section{REFERENCES}

[1] J. K. Shultis, and R.E. Faw, "Radiation Shielding Technology," Health Physics, vol. 88, no.4, pp. 297-300, April 2005.

[2] I. Carlomagno, "Synchrotron X-rays Methodologies for Aerospace Materials," 5th IEEE International Workshop on, pp.554-558, 2018.

[3] J. Viegas, L.A. Silva, A. M. S. Batista, C. A. Furtado, J. P. Nascimento, and L. O. Faria, "Increased X-ray Attenuation Efficiency of GrapheneBased Nanocomposite," Industrial \& Engineering Chemistry Research, vol. 40, no. 30, pp. 30-40, July 2016.

[4] Z. Wang, J. Luo, and G. L. Zhao, "Dielectric and Microwave Attenuation Properties of Graphene Nanoplatelet-Epoxy Composites," AIP Advances, vol. 4, pp. 1-10, January 2014.

[5] D. R. McAlister, "Gamma Ray Attenuation Properties of Common Shielding Materials", Ph.D. dissertation, PG Research Foundation, Inc. University Lane Lisle, USA, 2018.

[6] L. M. Warren, A. Mackenzie, D. R. Dance, and K. C. Young, "Comparison of the X-ray Attenuation Properties of Breast Calcifications, Aluminum, Hydroxyapatite and Calcium Oxalate," Physics in Medicine \& Biology, vol. 58, no. 7, pp. 24-30,July 2013. 
[7] T. Fujimori, S. Tsuruoka, B. Fugetsu, S. Maruyama, A. Tanioka, M. Terrones, M.S. Dresselhaus, M. Endo, and K. Kaneko, "Outstanding X-ray Shielding Effects of Carbon Nanotubes," Material Express, vol. 4, pp. 40-50, 2011.

[8] L.V.K.M. Tais, "The Effects of a Carbon Fiber Table Top on Radiation Dose and Image Quality During Fluoroscopy", M.Sc. dissertation, Dept. of Medical Engineering Advanced Level Royal Institute of Technology, England, 2014.

[9] J. P. McCaffrey, H. Shen, B. Downton, and E. M. Hing, "Radiation Attenuation by Lead and Non-Lead Materials used in Radiation Shielding Garments," Medical Physics, vol. 34, no. 2, pp. 530-535, February 2007.

[10] IAEA/WHO, Radiation Protection in Medicine Setting the Scene for the Next Decade, Proceedings of an International Conference 3-7 December 2012 Bonn, Germany, 31-35, 2012.

[11] K. Yue, W. Luo, X. Dong, C. Wang, G. Wu, M. Jiang, and Y. Zha, "A New Lead-Free Radiation Shielding Material for Radiotherapy," Radiation Protection Dosimetry, vol. 133, pp. 256-260, June 2009.

[12] P. Szajerski, M. Zaborski, H. Bem, W. Baryn, and E. Kusiak, "Optimization of the Heavy Metal (Bi-W-Gd-Sb) Concentrations in the Elastomeric Shields for Computer Tomography (CT), "Journal of Radioanalytical and Nuclear Chemistry, vol. 300, pp. 385-391, January 2014.

[13] S. Asai, K. Miyoshi, and K. Saito, "Modification of a Porous Sheet (MAPS) for the High-Performance Solid-Phase Extraction of Trace and Ultratrace Elements by Radiation-Induced Graft Polymerization," Analytical Science, vol. 26, pp. 649-658, April 2010.

[14] S.C. Kim, J.R. Choi, and B.K. Jeon," Physical Analysis of the Shielding Capacity for a Lightweight Apron Designed for Shielding Low Intensity Scattering X-rays," Science Report, vol. 6, pp. 14-30, October 2016.

[15] J.P. Yang, Z.K. Chen, G. Yang, S.Y. Fu, and L. Ye, "Simultaneous Improvements in the Cryogenic Tensile Strength, Ductility and Impact Strength of Epoxy Resins by a Hyperbranched Polymer," Polymer, vol. 49, pp. 3168-3175, December 2008.

[16] R. Mirji, and B. Lobo, "Radiation Shielding Materials: A Brief Review on Methods, Scope and Significance," Proc. National Conference on 'Advances in VLSI and Microelectronics', pp. 96-100, $27^{\text {th }}$ January 2017.

[17] A. Ribeiro, O. Husson, N. Drey, I. Murray, K. May, J. Thurston, and W. Oyen, "Ionising Radiation Exposure from Medical Imaging: A Review of Patient's (un) Awareness," Radiography, vol. 26, pp. 25-30, November 2020.

[18] O. Klein, and Y. Nishina, "The Scattering of Light by Free Electrons according to Dirac's New Relativistic Dynamics," Nature, vol. 122, pp. 398-400, August 1928.

[19] F. A. Scannavino, and P. E. Cruvinel, "A Graphical Tool for an Analytical Approach of Scattering Photons by the Compton Effect," Nuclear Instruments and Methods in Physics Research A, vol. 674, pp. 28-38, October 2012.

[20] P. Eisenberger, and P.M. Platzman, "Compton Scattering of X-rays from Bound Electrons," Physics Review A, vol. 2, pp. 415-420, March 1970.

[21] M. Puska, and R. Nieminen, "Theory of Positrons in Solids and on Solid Surfaces," Review of Modern Physics, vol. 66, pp. 841-850, November 1994.

[22] O. Klein, and T. Nishina, "Uber die Streuung von Strahlung durch freie Elektronen nach " der neuen relativistischen Quantendynamik von Dirac (in German)," Zeitschrift Physik, vol. 52, pp. 853-860, July 1929.

[23] H. Seo, "Experimental Performance of Double-Scattering Compton Camera with Anthropomorphic Phantom," JINST, vol. 6, pp. 1-10, September 2011.

[24] H. Kleinert, "Particles and Quantum Fields", World Scientific, Singapore, May 1996.

[25] J. H. Hubbell, "Review of Photon Interaction Cross Section Data in the Medical and Biological Context," Physics Medicine and Biology, vol. 44, pp. 1-22, August 1999.

[26] I. Hossain, N. A. B. Azmi, E. Hoque, and K. K. Viswanathan, "Compton Scattering of $662 \mathrm{keV}$ Gamma Rays Proposed by KleinNishina Formula," Scientific Research and Essays, vol. 6, no. 30, pp. 6312-6316, December 2011.

[27] C.V. Bowen, "Analytic 3D Scatier Correction in Positron Tomography Using the Klein-Nishina Equation," M.Sc. dissertation, Dept. of McMaster University, November 1994.

[28] F. H. Attix, "Introduction to Radiological Physics and Radiation Dosimetry: Chapter 7," Jhon Wiley \& Son, New York, Singapore, Toranto, 2015.

[29] S. Chen, and M. Kotlarchyk, "Interaction of Photons and Neutrons with Matter," Second ed., World Scientific, New Jersey, 1999.

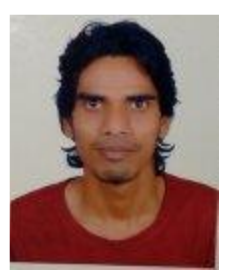

Mr. Saddam Husain Dhobi pursed B. Sc., \& M. Sc., in Physical Science from Tribhuvan University, Kathmandu-44600, Nepal 2016, \& 2020 . He is currently working as Sr. Researcher in Innovative Ghar Nepal Laboratory and Robotics Academy of Nepal, Lalitpur-44700, Nepal since 2013. He has published more than 30 research papers in reputed international journals including Thomson Reuters (SCI \& Web of Science), it's also available online. His main research work focuses on electron and photon properties beyond the general properties, Data Analytics, Virology, Nuclear Physics, and Computational Intelligence based education. He has 4 years of research experience.

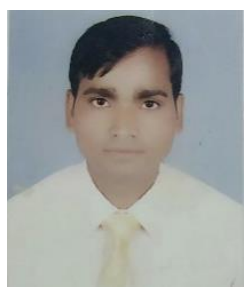

Mr. Sanotsh Kumar Das purses B.Sc. and M.Sc. in Physics Science from Science from Tribhuvan University, Kathmandu-44600, and Nepal. He is permanent Faculty member of Tribhuvan University at Physics Department. His Field of intrest is to study of electron, Photon, Quantum Mechanics and Statistical Mechanics.

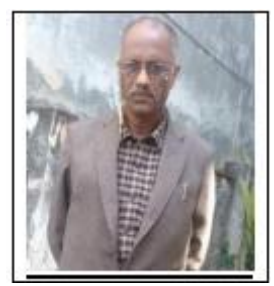

Mr. Kishori Yadav pursed B. Sc. in Physical Science from L.N. Mithila Univeristy Darbhanga, India in 1983 and M.Sc. from Tribhuvan University, Kathmandu-44600, and Nepal in 1992. He was awarwd Ph.D. 2019. He is currently working as Associate Prof. at Department of Physics, Tribhuvan University, Kathmandu44600, and Nepal. He has also published more than 9 research papers in reputed international journals including Thomson Reuters (SCI \& Web of Science), it's also available online. 